piteliali di alto grado.

Metodologia. In 138 pazienti che presentano displasia moderata o grave all'esame istologico su biopsia cervicale, è stata eseguita la ricerca dell'HPV su prelievo endocervicale utilizzando la reazione a catena della polimerasi (PCR) con primers specifici per la regione L1 e della regione E6-e E7 prima, 3 e 6 mesi dopo la conizzazione.

Risultati. Prima della conizzazione il 92\% (127/138) dei pazienti sono risultati positivi alla test HPV di cui il $75 \%$ ad alto rischio oncogeno (95/138), 16 ancora positive dopo 6 mesi. La malattia residua si è riscontrata in 13 casi di cui 10 HPV positivi con un valore predittivo positivo del $77 \%$ (10/13). Durante un periodo medio di 18 mesi di follow-up 3 pazienti hanno presentato una recidiva e sono risultati HPV positivi a tutti i controlli con un valore predittivo positivo del $100 \%(3 / 3)$

Conclusioni. I dati riportati evidenziano che il test HPV: 1) permette, insieme al pap-test, di selezionare le donne che hanno un rischio maggiore di malattia residua/recidiva e cancro della cervice dopo il trattamento delle lesioni di alto grado: 1) permette, con un VPP del 100\% nelle recidive, di ridurre la frequenza del follow-up in quei pazienti che hanno il test HPV negativo dopo trattamento con un miglior rapporto costo /beneficio.

\title{
203
}

\section{TEST HPV E GESTIONE DI MALATTIA \\ RESIDUA E RECIDIVE IN DONNE TRATTATE PER LESIONI INTRAEPITELIALI DI ALTO GRADO}

\author{
Bianchi L., Venturi C., Papucci A., Apicella P.
}

Ospedale di Pescia, "SS Cosma e Damiano", ASL 3 Pistoia, Zona della Val di Nievole, Via Cesare Battisti 2, 51017 Pescia (PT).

L'evidenza che l'infezione persistente della cervice uterina da parte di ceppi HPV ad alto rischio oncogeno (HRHPV:16, 18, 31, 33) sia la principale causa dello sviluppo del cancro della cervice uterina ha portato ad estendere la valutazione dell'utilità della ricerca dell'HPV anche al follow-up di donne trattate per lesioni di alto grado al pap test.

Obiettivi. Scopo di questo studio è valutare l'utilità del test HPV nel selezionare donne con un rischio più elevato di malattia residua/recidiva in donne trattate per lesioni intrae- 